\title{
ANALYSIS OF TWO-LEVEL METHOD FOR ANISOTROPIC DIFFUSION EQUATIONS ON ALIGNED AND NON-ALIGNED GRIDS
}

\author{
GUOZHU YU, JINCHAO XU, AND LUDMIL T. ZIKATANOV
}

\begin{abstract}
This paper is devoted to the multigrid convergence analysis for the linear systems arising from the conforming linear finite element discretization of the second order elliptic equations with anisotropic diffusion. The multigrid convergence behavior is known to strongly depend on whether the discretization grid is aligned or non-aligned with the anisotropic direction and analyses in the paper will be mainly focused on two-level algorithms. For an aligned grid case, a lower bound is given for point-wise smoother which shows deterioration of convergence rate. In both aligned and non-aligned cases we show that for a specially designed block smoother the convergence is uniform with respect to both anisotropy ratio and mesh size in the energy norm. The analysis is complemented with numerical experiments which confirm the theoretical results.
\end{abstract}

\section{INTRODUCTION}

In this paper we will study multilevel methods for anisotropic partial differential equations (PDEs) by finite element (FE) methods and in particular we will analyze the convergence behavior of some methods for anisotropic diffusion equations on grids that are either aligned or non-aligned with the anisotropy.

There are already many convergence results in the literature for multilevel method on anisotropic problems when the underlying FE grid is aligned with the anisotropy direction. The case of constant anisotropy was considered by Stevenson [1, 2], who established a uniform convergence of the $\mathrm{V}$-cycle multigrid methods. Main tools in his analysis are the so called classical smoothing and approximation properties (see Hackbusch [3]). The case of "mildly" varying anisotropy was analyzed in a work by Bramble and Zhang [4. Using a different theoretical framework developed in Bramble, Pasciak, Wang and Xu [5] and $\mathrm{Xu}$ [6], Neuss [7] also showed uniform convergence of the V-cycle algorithm for anisotropic diffusion problem. More recently, $\mathrm{Wu}$, Chen, Xie and $\mathrm{Xu}$ [8] analyzed V-cycle multigrid with line smoother and standard coarsening, and V-cycle multigrid with point Gauss-Seidel smoother and semi-coarsening at the same time, and they were able to prove convergence under weaker assumptions on the regularity of the solution of the underlying PDE. Another technique, based on tensor product type subspace splittings and a semi-coarsening was proposed and analyzed by Griebel and Oswald [9]. They have shown uniform and optimal condition number bounds for multilevel additive preconditioners.

Date: August 23, 2021.

1991 Mathematics Subject Classification. 65F10, 65N20, 65N30.

Key words and phrases. anisotropic problem, finite element discretization, iterative methods, two-level methods.

This work was completed while the first author was visiting the Center for Computational Mathematics and Applications (CCMA) at Penn State. Thanks go to the CCMA at PSU for the support of this work. The work of the third author is supported in part by the National Science Foundation, DMS-0810982, and OCI-0749202. 
The aforementioned theoretical convergence results on multigrid methods for the anisotropic diffusion equations are, however, all carried out under one main assumption that the anisotropy direction is aligned with the mesh. But such an assumption is not always satisfied in practice. In this paper, we will make an attempt to develop uniform convergence theory in certain cases when this aligned grid assumption is not satisfied. More specifically, we will study the problem (2.1) in the case that $\Omega$ is a square domain triangulated by uniform grids that is rotated by an angle $\omega \in[0, \pi]$. We have grids that are not aligned with anisotropy except for special cases that $\omega=0, \omega=\frac{\pi}{2}$ or $\omega=\frac{3 \pi}{4}$.

For this special class of domains and grids, we will design a two-level method and prove its uniform convergence (with respect to both anisotropy and mesh size). We are not yet able to extend our theoretical analysis neither to multilevel (more than two levels) case, nor to more general anisotropic problems. We hope however that the analysis presented here, even though in a special case, can be extended to handle more general anisotropic problems.

We would like to point out that our work was partially motivated by some recent theoretical results for nearly singular problems (see [10]). Indeed, anisotropic diffusion equation gets more nearly singular when anisotropy gets smaller. Techniques such as line smoother or semicoarsening correspond in some way to space splittings of the so called near kernel components of the anisotropic diffusion problem. We refer to [10] for description of such splittings.

The rest of this paper is organized as follows. In Section 2 we introduce the notation and preliminaries. In Section 3 we state the main result. We then prove several stability and interpolation estimates for coarse grid interpolant in Section 4 and for the fine grid interpolant in Section 5. In section 6 we prove the main theorem, already stated in section 3 . Numerical experiments, which verify the theory are given in section 7 .

\section{Preliminaries and notation}

Consider the anisotropic diffusion equation on a square domain $\Omega \subset \mathbb{R}^{2}$ :

$$
\left\{\begin{aligned}
-u_{x x}-\epsilon u_{y y}=f, & \text { in } \Omega, \\
u=0, & \text { on } \partial \Omega,
\end{aligned}\right.
$$

where $\epsilon>0$ is a constant. We are interested in the case when $\epsilon \rightarrow 0$. The weak formulation of 2.1 is: Find $u \in H_{0}^{1}(\Omega)$ such that

$$
a(u, v)=(f, v), \quad \forall v \in H_{0}^{1}(\Omega),
$$

where

$$
a(u, v)=\int_{\Omega}\left(\partial_{x} u \partial_{x} v+\epsilon \partial_{y} u \partial_{y} v\right) d x d y, \quad \text { and } \quad(f, v)=\int_{\Omega}(f v) d x d y .
$$

We consider family of computational domains $\Omega$ obtained by rotations of a fixed domain $\Omega_{0}=(-1,1)^{2}$ around the origin. The angles of rotation are denoted by $\omega$ and we consider $\omega \in$ $[0, \pi]$, since this covers all the possible cases of alignment (non-alignment) of the anisotropy and the FE grid.

We assume that we have initial triangulation $\mathcal{T}_{0}$ of the domain $\Omega_{0}$, obtained by dividing $\Omega_{0}$ into $N \times N$ equal squares and then dividing every square into two triangles. Then we rotate $\Omega_{0}$ around the origin to obtain the computational domain $\Omega$ and its triangulation $\mathcal{T}_{h}$. The finite element function space associated with $\Omega$ and $\mathcal{T}_{h}$ will be the space of piece-wise continuous linear functions with respect to $\mathcal{T}_{h}$ and we denote this space by $V_{h}$. One may see three such domains shown in Figure 2.1. In such setting one case of grid aligned anisotropy corresponds to $\Omega=\Omega_{0}$, or equivalently, $\omega=0$. 

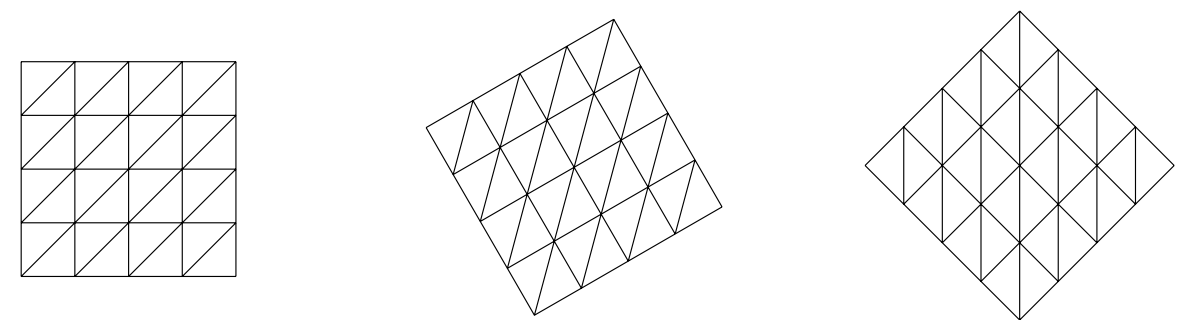

Figure 2.1. The domains from left to right are corresponding to $\omega=0, \omega=\frac{\pi}{6}$ and $\omega=\frac{\pi}{4}$ respectively.

Given a coarse mesh $\mathcal{T}_{H}$, assume the fine mesh $\mathcal{T}_{h}$ is obtained from $\mathcal{T}_{H}$ by splitting each of the triangles in the triangulation $\mathcal{T}_{H}$ into four congruent triangles. One clearly then has $h=H / 2$. The spaces of continuous piece-wise linear functions corresponding to the partitions $\mathcal{T}_{h}$ and $\mathcal{T}_{H}$ are denoted by $V_{h}$ and $V_{H}$. As it is customary $I_{h}, I_{H}$ will denote the nodal interpolation operators mapping to $V_{h}$ and $V_{H}$ respectively.

For the analysis of the two-level method, we introduce partition of unity $\left\{\theta_{i}(y)\right\}_{i=1}^{L}$, where $L=\left[\frac{y_{\max }-y_{\min }}{h}\right]$, with $y_{\max }=\max _{(x, y) \in \Omega}\{y\}, y_{\min }=\min _{(x, y) \in \Omega}\{y\}$. We define $\theta_{i}$ as follows:

$$
\theta_{i}(y)= \begin{cases}\frac{\left(y-y_{\min }\right)-(i-1) h}{h}, & (i-1) h \leq y-y_{\min } \leq i h, \\ \frac{(i+1) h-\left(y-y_{\min }\right)}{h}, & i h \leq y-y_{\min } \leq(i+1) h \\ 0, & \text { other. }\end{cases}
$$

Note that each $\theta_{i}$ is piece-wise linear in the $y$ variable and is constant in $x$. Moreover, each $\theta_{i}$ is supported in the $i$-th $\operatorname{strip}(i-1) h \leq y-y_{\text {min }} \leq(i+1) h$.

Denote the set of triangles in $\mathcal{T}_{h}$ including nodes in the $i$-th strip by $\mathcal{T}_{i}$, and Let

$$
\begin{gathered}
\Omega_{i}=\bigcup_{\tau \in \mathcal{T}_{i}} \tau, \\
V_{i}=\left\{v \in V_{h}, \operatorname{supp} v \subseteq \bar{\Omega}_{i}\right\},
\end{gathered}
$$

then the two-level method with line smoother $V_{i}(1 \leq i \leq L)$ and coarse grid $V_{H}$ can be written as

$$
V_{h}=\sum_{i=1}^{L} V_{i}+V_{H}
$$

Let $K$ be a triangle with vertices $\left\{\left(x_{i}, y_{i}\right)\right\}_{i=1}^{3}$, which we assume ordered counter-clockwise. For a given edge $E \in \partial K$, with

$$
E=\left(\left(x_{i}, y_{i}\right),\left(x_{j}, y_{j}\right)\right), \quad j=1+\bmod (i, 3),
$$

we denote

$$
\delta_{E}^{K} y=\frac{1}{2|K|}\left(y_{j}-y_{i}\right)
$$

we also denote with $\left(x_{E}, y_{E}\right)$ the coordinates of the vertex of $K$ which is opposite to $E$. In another word, if $E=\left(\left(x_{i}, y_{i}\right),\left(x_{j}, y_{j}\right)\right)$ then $\left(x_{E}, y_{E}\right)=\left(x_{k}, y_{k}\right)$, where $k \neq i$ and $k \neq j$. Let 
$v$ be a linear function on $K$. If we set $v_{E}^{K}=v\left(x_{E}, y_{E}\right)$, then, it is easy to check that

$$
\left.\frac{\partial v}{\partial x}\right|_{K}=\sum_{E \in \partial K}\left(\delta_{E}^{K} y\right) v_{E}^{K} .
$$

\section{Convergence of the tWo-Level method}

We will first prove that even in the aligned case the most common point-wise smoothers will result in two-level method whose convergence deteriorates when $\epsilon$ tends to zero in equation (2.1). The result is as follows.

Theorem 3.1 (Lower bound and deterioration of the convergence rate). In case of grid aligned anisotropy (i.e. $\omega=0, \frac{\pi}{2}, \frac{3 \pi}{4}$ ) the energy norm of the error propagation operator corresponding to the two-level iteration with coarse space $V_{H}$ and point-wise Gauss-Seidel smoother can be bounded below as follows:

$$
\left\|E_{T L}\right\|_{a}^{2} \geq 1-C\left(\epsilon+h^{2}\right)
$$

with constant $C$ independent of $\epsilon$ and $h$.

This result follows from the following two-level convergence identity (proof can be found in [11, Lemma 2.3]):

Lemma 3.2. The following relation holds for the two-level error propagation operator $E_{T L}=$ $(I-T)\left(I-P_{H}\right)$ :

$$
\left\|E_{T L}\right\|_{a}^{2}=1-\frac{1}{K} \quad \text { where } \quad K=\sup _{v \in V} \frac{\left\|\left(I-\Pi_{*}\right) v\right\|_{*}^{2}}{\|v\|_{a}^{2}},
$$

where $\|v\|_{*}^{2}=\inf _{\sum_{i} v_{i}=v} \sum_{k}\left\|v_{k}\right\|_{a}^{2}$ and $\Pi_{*}$ is an $(\cdot, \cdot)_{*}$-orthogonal projection on $V_{H}$.

Proof of Theorem 3.1. From the Lemma 3.2 we can immediately see that to prove the estimate (3.1) we need to show that

$$
K=\sup _{v \in V_{h}} \frac{\left\|\left(I-\Pi_{*}\right) v\right\|_{*}^{2}}{\|v\|_{a}^{2}} \gtrsim \frac{1}{\epsilon+h^{2}},
$$

here the quantity $K$ is the same as in Lemma 3.2. From the proof of [11, Theorem 4.5], we also know that

$$
K \gtrsim h^{-2} \sup _{v \in V_{h}} \frac{\left\|\left(I-Q_{H}\right) v\right\|^{2}}{\|v\|_{a}^{2}},
$$

where $Q_{H}$ is the $(\cdot, \cdot)$ orthogonal projection on $V_{H}$. In the case of angle of rotation $\omega=0$, the computational domain is $\Omega=\Omega_{0}=(-1,1)^{2}$. We assume $h=1 / n$ ( $n$ is even) and consider the $2 n \times 2 n$ partition with vertices $\left(x_{j}, y_{k}\right), x_{j}=j h$ and $y_{k}=k h, j=-n, \cdots, n, k=$ $-n, \cdots, n$, then the corresponding coarse gird is with vertices $\left(x_{2 j}, y_{2 k}\right), j=-n / 2, \cdots, n / 2$, $k=-n / 2, \cdots, n / 2$.

For any given $v \in V_{h}$, since

$$
\|v\|^{2} \simeq h^{2} \sum_{j=-n}^{n} \sum_{k=-n}^{n} v^{2}\left(x_{j}, y_{k}\right),
$$


and

$$
\left\|I_{H} v\right\|^{2} \simeq H^{2} \sum_{j=-n / 2}^{n / 2} \sum_{k=-n / 2}^{n / 2} v^{2}\left(x_{2 j}, y_{2 k}\right)
$$

then the interpolation $I_{H}$ is stable in the $L_{2}$ norm, i.e. $\left\|I_{H} v\right\| \lesssim\|v\|$, for all $v \in V_{h}$, provided that $\frac{H}{h} \lesssim 1$. Now consider a function $v_{0} \in V_{h}$, supported in the closure of $(-1,1) \times(0,2 h)$ and defined as

$$
v_{0}\left(x_{j}, y_{1}\right)=v_{0}\left(x_{j}, h\right)=1-|j| h, j=-n, \cdots, n,
$$

and $v_{0}$ is 0 at any other vertex. Note that

$$
I_{H} v_{0}=0 .
$$

From the stability of $I_{H}$ in the $L_{2}$ norm, which we have just shown we get:

$$
\begin{aligned}
\left\|I_{H}\left(I-Q_{H}\right) v_{0}\right\|^{2} & \lesssim\left\|\left(I-Q_{H}\right) v_{0}\right\|^{2}, \\
\left\|\left(I-I_{H}\right)\left(I-Q_{H}\right) v_{0}\right\|^{2} & \lesssim\left\|\left(I-Q_{H}\right) v_{0}\right\|^{2} .
\end{aligned}
$$

Using these estimates and the fact that $I_{H} Q_{H}=Q_{H}$ then gives

$$
\begin{aligned}
\left\|\left(I-Q_{H}\right) v_{0}\right\|^{2} & \gtrsim\left\|I_{H}\left(I-Q_{H}\right) v_{0}\right\|^{2}+\left\|\left(I-I_{H}\right)\left(I-Q_{H}\right) v_{0}\right\|^{2} \\
& =\left\|\left(I_{H}-Q_{H}\right) v_{0}\right\|^{2}+\left\|\left(I-I_{H}\right) v_{0}\right\|^{2} \\
& =\left\|Q_{H} v_{0}\right\|^{2}+\left\|v_{0}\right\|^{2} \\
& \geq\left\|v_{0}\right\|^{2} .
\end{aligned}
$$

So

$$
K \gtrsim h^{-2} \sup _{v \in V_{h}} \frac{\left\|\left(I-Q_{H}\right) v\right\|^{2}}{\|v\|_{a}^{2}} \gtrsim h^{-2} \frac{\left\|\left(I-Q_{H}\right) v_{0}\right\|^{2}}{\left\|v_{0}\right\|_{a}^{2}} \gtrsim h^{-2} \frac{\left\|v_{0}\right\|^{2}}{\left\|v_{0}\right\|_{a}^{2}} .
$$

Since

$$
\left\|v_{0}\right\|^{2} \simeq h^{2} \sum_{j=-n}^{n} v_{0}^{2}\left(x_{j}, h\right) \simeq h^{2} \sum_{j=0}^{n}(1-j h)^{2}=h^{4} \sum_{j=0}^{n} j^{2} \simeq h^{4} n^{3} \simeq h
$$

and

$$
\left\|v_{0}\right\|_{a}^{2}=\left\|\partial_{x} v_{0}\right\|^{2}+\epsilon\left\|\partial_{y} v_{0}\right\|^{2} \simeq h+\epsilon / h
$$

then

$$
K \gtrsim h^{-2} \frac{\left\|v_{0}\right\|^{2}}{\left\|v_{0}\right\|_{a}^{2}} \gtrsim h^{-2} \cdot h \cdot \frac{h}{\epsilon+h^{2}}=\frac{1}{\epsilon+h^{2}}
$$

The above results show that in case of a grid that is aligned with the anisotropy direction the convergence of a standard two-level method (point-wise smoother and standard coarsening) will deteriorate. One easily sees that for $\epsilon \leq h^{2}$ we get a poor convergence rate (no better than $1-\mathcal{O}\left(h^{2}\right)$ ).

However, the next result shows that when the grid is not aligned with the anisotropy direction (e.g., angle of rotation $\omega=\pi / 4$ ) the lower bound given in Lemma 3.1 does not apply and the standard two-level method is uniformly convergent in this case. 
Lemma 3.3. Assume that $\Omega$ is obtained from $\Omega_{0}$ by a rotation with angle of rotation $\omega=\frac{\pi}{4}$. Then the error propagation operator corresponding to the two-level iteration with coarse space $V_{H}$ and point-wise Gauss-Seidel smoother is a uniform contraction in the energy norm. In fact, we have the estimate

$$
K=\sup _{v \in V_{h}} \frac{\left\|\left(I-\Pi_{*}\right) v\right\|_{*}^{2}}{\|v\|_{a}^{2}} \leq C
$$

and so

$$
\left\|E_{T L}\right\|_{a}^{2} \leq 1-\frac{1}{C}
$$

with constant $C$ independent of $\epsilon$ and $h$.

Proof. The proof follows the same lines as the proof of Theorem 3.4, which will be given in the Section 6.

Let us remark here that for decreasing values of the angle of rotation (i.e. decreasing $\omega$ from $\pi / 4$ to 0$)$ the convergence rate $\left\|E_{T L}\right\|_{a}$ of a two-level method with point-wise smoother deteriorates as the angle of rotation becomes smaller.

From the above considerations, it is clear that even in the case of aligned anisotropy one needs to use a special smoother or coarsening strategy in order to achieve uniform convergence. Our analysis shows that the line smoother or more generally a block smoother with blocks consisting of degrees of freedom along the anisotropy results in a uniformly convergent method. The Theorem below provides a uniform estimate on the convergence rate of the error propagation operator and is the main result in this paper.

Theorem 3.4. For any angle of rotation $\omega \in[0, \pi]$, the two-level iteration with coarse space $V_{H}$ and line (block) Gauss-Seidel smoother is a uniformly convergent method. In fact, we have

$$
\left\|E_{T L}\right\|_{a}^{2} \leq 1-\frac{1}{C}
$$

with constant $C$ independent of $\epsilon$ and $h$.

The proof of this theorem is postponed to Section 6. The result follows from the stability and interpolation estimates that are given in Section 4 and Section 5 and Lemma 3.2 .

\section{Stability OF THE COARSE GRID INTERPOLANT}

In this section we prove the stability of the coarse grid interpolant.

In what follows, given a triangle $K \in \mathcal{T}_{H}\left(K=\bigcup_{l=1}^{4} K_{l}\right.$ with $\left.K_{l} \in \mathcal{T}_{h}\right)$ as shown in the Figure 4.1, we shall frequently use the following equalities

$$
\begin{aligned}
& y_{2}-y_{6}=y_{6}-y_{1}=y_{4}-y_{5}, \\
& y_{3}-y_{4}=y_{4}-y_{2}=y_{5}-y_{6}, \\
& y_{1}-y_{5}=y_{5}-y_{1}=y_{6}-y_{4}, \\
& |K|=4\left|K_{l}\right|, \quad l=1 \ldots 4 .
\end{aligned}
$$

Proposition 4.1. For any $v \in V_{h}$, we have the following relation:

$$
\left.\frac{\partial\left(I_{H} v\right)}{\partial x}\right|_{K}=\frac{1}{2}\left(\left.\sum_{l=1}^{3} \frac{\partial v}{\partial x}\right|_{K_{l}}-\left.\frac{\partial v}{\partial x}\right|_{K_{4}}\right) .
$$




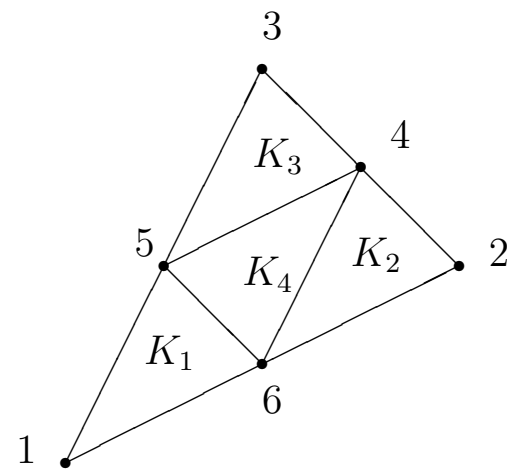

Figure 4.1. A refinement of a coarse element $K$

Proof. From (2.5) with $I_{H} v$ instead of $v$, we have

$$
\left.\frac{\partial\left(I_{H} v\right)}{\partial x}\right|_{K}=\sum_{E \in \partial K}\left(\delta_{E}^{K} y\right) v_{E}^{K},
$$

and from 2.5 with $K_{l}$ instead of $K$ for $l=1, \ldots, 4$, we have

$$
\left.\frac{\partial v}{\partial x}\right|_{K_{l}}=\sum_{E \in \partial K_{l}}\left(\delta_{E}^{K_{l}} y\right) v_{E}^{K_{l}} .
$$

Combine the above two equations and (4.1), it is immediate to verify the result.

We are now ready to prove our first stability estimate. Since we have anisotropic diffusion problem in hand, we need to estimate separately $\left\|\frac{\partial\left(I_{H} v\right)}{\partial x}\right\|_{0}$ and $\left\|\frac{\partial\left(I_{H} v\right)}{\partial y}\right\|_{0}$, which is done in the next Lemma.

Lemma 4.2. For any $v \in V_{h}$, we have $\left\|\frac{\partial\left(I_{H} v\right)}{\partial x}\right\|_{0}^{2} \leq 4\left\|\frac{\partial v}{\partial x}\right\|_{0}^{2}$, and $\left\|\frac{\partial\left(I_{H} v\right)}{\partial y}\right\|_{0}^{2} \leq 4\left\|\frac{\partial v}{\partial y}\right\|_{0}^{2}$.

Proof. We only need to prove this estimate locally for any $K \in \mathcal{T}_{H}$. So we fix $K \in \mathcal{T}_{H}$ and we would like to show that $\left\|\frac{\partial\left(I_{H} v\right)}{\partial x}\right\|_{0, K}^{2} \leq 4\left\|\frac{\partial v}{\partial x}\right\|_{0, K}^{2}$.

From 4.2 , for the $L_{2}$ norm $\left\|\frac{\partial\left(I_{H} v\right)}{\partial x}\right\|_{0, K}^{2}$ we have

$$
\begin{aligned}
\left\|\frac{\partial\left(I_{H} v\right)}{\partial x}\right\|_{0, K}^{2} & =\frac{|K|}{4}\left(\left.\sum_{l=1}^{3} \frac{\partial v}{\partial x}\right|_{K_{l}}-\left.\frac{\partial v}{\partial x}\right|_{K_{4}}\right)^{2} \\
& \leq|K| \sum_{l=1}^{4}\left(\left.\frac{\partial v}{\partial x}\right|_{K_{l}}\right)^{2}=4 \sum_{l=1}^{4}\left|K_{l}\right|\left(\left.\frac{\partial v}{\partial x}\right|_{K_{l}}\right)^{2} \\
& =4 \sum_{l=1}^{4}\left\|\frac{\partial v}{\partial x}\right\|_{0, K_{l}}^{2}=4\left\|\frac{\partial v}{\partial x}\right\|_{0, K}^{2} .
\end{aligned}
$$

Summing over all the elements then gives $\left\|\frac{\partial\left(I_{H} v\right)}{\partial x}\right\|_{0}^{2} \leq 4\left\|\frac{\partial v}{\partial x}\right\|_{0}^{2}$. In a similar fashion we can prove that $\left\|\frac{\partial\left(I_{H} v\right)}{\partial y}\right\|_{0}^{2} \leq 4\left\|\frac{\partial v}{\partial y}\right\|_{0}^{2}$, and the proof of the lemma is complete.

As a consequence, we have the following approximation result for the coarse grid interpolant. 
Lemma 4.3. For any $v \in V_{h}$, we have

$$
\left\|\frac{\partial\left(v-I_{H} v\right)}{\partial x}\right\|_{0}^{2} \lesssim\left\|\frac{\partial v}{\partial x}\right\|_{0}^{2}, \quad\left\|\frac{\partial\left(v-I_{H} v\right)}{\partial y}\right\|_{0}^{2} \lesssim\left\|\frac{\partial v}{\partial y}\right\|_{0}^{2},
$$

and

$$
\left\|v-I_{H} v\right\|_{0}^{2} \lesssim h^{2}|v|_{1}^{2}
$$

Proof. The first two estimates follow from the inequalities given in Lemma 4.2. The third estimate can be found in [12, Lemma 4.4].

Remark 4.4. In fact, in the proof of Lemma 4.2, there is no any requirement for the partition. The result is true for partition $\mathcal{T}_{h}$ obtaining from regular refinement of any given partition $\mathcal{T}_{H}$. So is the Lemma 4.3 .

\section{Stability estimates ON The Fine GRID}

In this section we give estimates on the stability of the partition of unity introduced in Section 2, equation (2.3). In what follows, to avoid proliferation of indicies, we will omit the subscript $i$ and we will write $\theta$ instead of $\theta_{i}$.

For any given $K \in \mathcal{T}_{H}\left(K=\bigcup_{l=1}^{4} K_{l}\right.$ with $\left.K_{l} \in \mathcal{T}_{h}\right)$ shown in Figure 4.1, we label with 1, 2, and 3 the vertices of $K$, and 4,5 , and 6 the midpoints of $K$ (these are also vertices of $K_{4}$ ). The corresponding coordinates are denoted by $\left(x_{j}, y_{j}\right), 1 \leq j \leq 6$. Further, for a continuous function $v$, when there is no confusion, we write $v_{j}:=v\left(x_{j}, y_{j}\right)$.

In what follows we also denote

$$
E_{\min }=\arg \min _{E \in \partial K_{4}}\left\{\left|\delta_{E}^{K_{4}} y\right|\right\}, \quad \text { where } \delta_{E}^{K_{4}} y \text { is defined in (2.4). }
$$

Then $E_{\min }$ is related to the anisotropic direction. In fact, to indicate the dependence on the particular element, one may write $E_{\text {min }}^{K_{4}}$ instead of $E_{\text {min }}$, but for simplicity we have chosen to omit the superscript $K_{4}$. Furthermore, we may denote $E_{\min }^{\prime}=\arg \min _{E^{\prime} \in \partial K_{4}^{\prime}}\left\{\left|\delta_{E^{\prime}}^{K_{4}^{\prime}} y\right|\right\}$.

Let us now consider a function $w \in V_{h}$ vanishing at the coarse grid vertices, that is, $w$ satisfies $I_{H} w=0$. We have the following 4 cases on a fixed $K \in \mathcal{T}_{H}$ :

Case $0 . \theta$ is zero in $K_{4}$;

Case 1. $\theta$ is nonzero in $K_{4}$ and convex in $K_{4}$ (i.e. $\theta \neq 0$ a.e. in $K_{4}$ );

Case 2. $\theta$ is nonzero at only one of the vertices of $K_{4}$ and concave in $K_{4}$;

Case 3. $\theta$ is nonzero at exactly two of the vertices of $K_{4}$ and concave in $K_{4}$.

The rest of this section contains technical results and their proofs, which can be classified according to the cases above. To prove the stability estimates on the fine grid, we need to bound $\left\|\frac{\partial\left(I_{h}(\theta w)\right)}{\partial x}\right\|$.

- For the Case 0, there is nothing to prove, since in this case $I_{h}(\theta w)=0$.

- For the Case 1 the corresponding estimate is given in Lemma 5.2. In Case 1 we also need to assume quasi-uniformity of the mesh. We also note that Proposition 5.3 (for Case 1) contains an estimate which is later used in Case 2 and Case 3.

- The stability estimates in Case 2 and Case 3 are given in Lemmas 5.4 and 5.5 , respectively, under the assumption that $\mathcal{T}_{h}$ is a uniform partition. 
Remark 5.1. In summary, for uniform mesh we have proved the stability estimate in all cases. In addition, we have proved some of the results in more general case of unstructured, but quasi-uniform mesh (Case 1).

Lemma 5.2. Assume that $\theta \neq 0$ in $K_{4}$ and convex in $K_{4}$ (Case 1). Then

$$
\left\|\frac{\partial\left(I_{h}(\theta w)\right)}{\partial x}\right\|_{0, K}^{2} \lesssim\left\|\frac{\partial(\theta w)}{\partial x}\right\|_{0, K}^{2}
$$

Proof. Since $I_{H} w=0$ we obviously have that $I_{H}(\theta w)=0$ as well. From 4.2 in Proposition 4.1 with $v=I_{h}(\theta w)$ we obtain that

$$
\left.\sum_{l=1}^{3} \frac{\partial\left(I_{h}(\theta w)\right)}{\partial x}\right|_{K_{l}}-\left.\frac{\partial\left(I_{h}(\theta w)\right)}{\partial x}\right|_{K_{4}}=\left.2 \frac{\partial\left(I_{H}\left(I_{h}(\theta w)\right)\right)}{\partial x}\right|_{K}=\left.2 \frac{\partial\left(I_{H}(\theta w)\right)}{\partial x}\right|_{K}=0
$$

In addition, from 4.2 , with $v=w$ we have

$$
\left.\sum_{l=1}^{3} \frac{\partial w}{\partial x}\right|_{K_{l}}-\left.\frac{\partial w}{\partial x}\right|_{K_{4}}=\left.2 \frac{\partial\left(I_{H} w\right)}{\partial x}\right|_{K}=0
$$

Therefore, from 5.2 for the $L^{2}$ norm $\left\|\frac{\partial\left(I_{h}(\theta w)\right)}{\partial x}\right\|_{0, K}$ we have:

$$
\begin{aligned}
\left\|\frac{\partial\left(I_{h}(\theta w)\right)}{\partial x}\right\|_{0, K}^{2}= & \sum_{l=1}^{4}\left|K_{l}\right|\left(\left.\frac{\partial\left(I_{h}(\theta w)\right)}{\partial x}\right|_{K_{l}}\right)^{2} \approx \sum_{l=1}^{3}\left|K_{l}\right|\left(\left.\frac{\partial\left(I_{h}(\theta w)\right)}{\partial x}\right|_{K_{l}}\right)^{2} \\
\approx & \frac{1}{|K|}\left\{\left[\left(y_{4}-y_{6}\right) \theta_{6} w_{6}+\left(y_{5}-y_{4}\right) \theta_{5} w_{5}\right]^{2}\right. \\
& +\left[\left(y_{5}-y_{4}\right) \theta_{4} w_{4}+\left(y_{6}-y_{5}\right) \theta_{6} w_{6}\right]^{2} \\
& \left.+\left[\left(y_{6}-y_{5}\right) \theta_{5} w_{5}+\left(y_{4}-y_{6}\right) \theta_{4} w_{4}\right]^{2}\right\} .
\end{aligned}
$$

On the other hand, since $\theta$ is a convex function in $K_{4}$, and $\theta$ is supported in a $2 h$ width strip, $\theta$ should be convex in at least three of $K_{l}(l=1: 4)$.

For any $K_{l}$ in which $\theta$ is convex, we have

$$
\|\theta\|_{0, K_{l}}^{2} \gtrsim\left|K_{l}\right| \sum_{j=1}^{3} \theta^{2}\left(x_{j}^{K_{l}}, y_{j}^{K_{l}}\right)
$$

where $\left(x_{j}^{K_{l}}, y_{j}^{K_{l}}\right)$ denote the coordinates of the $j$-th vertex of element $K_{l}$ for $j=1: 3$. Since the mesh is quasi-uniform, we have that $\max _{j=1: 3}\left\{y_{j}^{K_{l}}\right\}-\min _{j=1: 3}\left\{y_{j}^{K_{l}}\right\} \gtrsim h$, and there exists at least one vertex $j_{0}$ such that $\theta\left(x_{j_{0}}, y_{j_{0}}\right)=\theta\left(y_{j_{0}}\right) \gtrsim 1$. Hence, if $\theta$ is a convex function in $K_{l}$, then $\|\theta\|_{0, K_{l}}^{2} \gtrsim\left|K_{l}\right|$, and we conclude that there are at least three elements $K_{l}$, where $\|\theta\|_{0, K_{l}}^{2} \gtrsim\left|K_{l}\right|$ holds. 
From this argument and $(5.3)$ we get

$$
\begin{gathered}
\left\|\frac{\partial(\theta w)}{\partial x}\right\|_{0, K}^{2}=\left\|\theta \frac{\partial w}{\partial x}\right\|_{0, K}^{2}=\sum_{l=1}^{4}\|\theta\|_{0, K_{l}}^{2}\left(\left.\frac{\partial w}{\partial x}\right|_{K_{l}}\right)^{2} \approx \sum_{l=1}^{3}\left|K_{l}\right|\left(\left.\frac{\partial w}{\partial x}\right|_{K_{l}}\right)^{2} \\
\approx \frac{1}{|K|}\left\{\left[\left(y_{4}-y_{6}\right) w_{6}+\left(y_{5}-y_{4}\right) w_{5}\right]^{2}\right. \\
+\left[\left(y_{5}-y_{4}\right) w_{4}+\left(y_{6}-y_{5}\right) w_{6}\right]^{2} \\
\left.+\left[\left(y_{6}-y_{5}\right) w_{5}+\left(y_{4}-y_{6}\right) w_{4}\right]^{2}\right\}
\end{gathered}
$$

Introducing now

$$
M=\left(\begin{array}{ccc}
0 & y_{5}-y_{4} & y_{4}-y_{6} \\
y_{5}-y_{4} & 0 & y_{6}-y_{5} \\
y_{4}-y_{6} & y_{6}-y_{5} & 0
\end{array}\right), \quad \Theta=\left(\begin{array}{ccc}
\theta_{4} & & \\
& \theta_{5} & \\
& & \theta_{6}
\end{array}\right)
$$

we rewrite $(5.4)$ as

$$
\left\|\frac{\partial\left(I_{h}(\theta w)\right)}{\partial x}\right\|_{0, K}^{2} \approx \frac{1}{|K|}\|M \Theta \boldsymbol{z}\|_{\ell_{2}}^{2}, \quad \boldsymbol{z}=\left(w_{4}, w_{5}, w_{6}\right)^{t}
$$

while (5.5) can be rewritten as

$$
\left\|\frac{\partial(\theta w)}{\partial x}\right\|_{0, K}^{2} \approx \frac{1}{|K|}\|M \boldsymbol{z}\|_{\ell_{2}}^{2}, \quad \boldsymbol{z}=\left(w_{4}, w_{5}, w_{6}\right)^{t} .
$$

Here, $\|\cdot\|_{\ell_{2}}$ is the usual Euclidean norm on $\mathbb{R}^{3}$.

To prove the estimate $\left\|\frac{\partial\left(I_{h}(\theta w)\right)}{\partial x}\right\|_{0, K}^{2} \lesssim\left\|\frac{\partial(\theta w)}{\partial x}\right\|_{0, K}^{2}$, we only need to show that

$$
\frac{1}{|K|}\|M \Theta \boldsymbol{z}\|_{\ell_{2}}^{2} \lesssim \frac{1}{|K|}\|M \boldsymbol{z}\|_{\ell_{2}}^{2} \quad \text { for all } \quad \boldsymbol{z} \in \mathbb{R}^{3} .
$$

Such an inequality is easy to get in the case of $\operatorname{det}(M)=0$, so we may assume $M$ is invertible (i.e. $\left.\operatorname{det}(M)=2\left(y_{6}-y_{5}\right)\left(y_{4}-y_{6}\right)\left(y_{5}-y_{4}\right) \neq 0\right)$. We then need a bound on the eigenvalues of $M^{-1} \Theta M^{2} \Theta M^{-1}=\left(M^{-1} \Theta M\right)\left(M^{-1} \Theta M\right)^{T}$. In fact, we only need to bound the entries of $M^{-1} \Theta M$ because all the norms of this $3 \times 3$ matrix are equivalent. Thus, if the entries of $M^{-1} \Theta M$ are bounded in absolute value, then the eigenvalues of $\left(M^{-1} \Theta M\right)\left(M^{-1} \Theta M\right)^{T}$ are bounded and consequently (5.6) holds.

Directly computing the inverse of $M$ gives

$$
M^{-1}=\frac{1}{\operatorname{det}(M)}\left(\begin{array}{ccc}
-\left(y_{6}-y_{5}\right)^{2} & \left(y_{6}-y_{5}\right)\left(y_{4}-y_{6}\right) & \left(y_{6}-y_{5}\right)\left(y_{5}-y_{4}\right) \\
\left(y_{4}-y_{6}\right)\left(y_{6}-y_{5}\right) & -\left(y_{4}-y_{6}\right)^{2} & \left(y_{4}-y_{6}\right)\left(y_{5}-y_{4}\right) \\
\left(y_{5}-y_{4}\right)\left(y_{6}-y_{5}\right) & \left(y_{5}-y_{4}\right)\left(y_{4}-y_{6}\right) & -\left(y_{5}-y_{4}\right)^{2}
\end{array}\right) .
$$

We then calculate $M^{-1} \Theta M$ to obtain that

$$
M^{-1} \Theta M=\frac{1}{2}\left(\begin{array}{ccc}
\theta_{5}+\theta_{6} & \frac{y_{6}-y_{5}}{y_{4}-y_{6}}\left(-\theta_{4}+\theta_{6}\right) & \frac{y_{6}-y_{5}}{y_{5}-y_{4}}\left(-\theta_{4}+\theta_{5}\right) \\
\frac{y_{4}-y_{6}}{y_{6}-y_{5}}\left(-\theta_{5}+\theta_{6}\right) & \theta_{4}+\theta_{6} & \frac{y_{4}-y_{6}}{y_{5}-y_{4}}\left(\theta_{4}-\theta_{5}\right) \\
\frac{y_{5}-y_{4}}{y_{6}-y_{5}}\left(\theta_{5}-\theta_{6}\right) & \frac{y_{5}-y_{4}}{y_{4}-y_{6}}\left(\theta_{4}-\theta_{6}\right) & \theta_{4}+\theta_{5}
\end{array}\right) .
$$

Since $\theta$ is convex in $K_{4}$, by the definition of $\theta$, it is easy to see that

$$
\begin{aligned}
&\left|\theta_{6}-\theta_{5}\right| \lesssim h^{-1}\left|y_{6}-y_{5}\right|, \\
&\left|\theta_{5}-\theta_{4}\right| \lesssim h^{-1}\left|y_{5}-y_{4}\right|,
\end{aligned}
$$




$$
\left|\theta_{4}-\theta_{6}\right| \lesssim h^{-1}\left|y_{4}-y_{6}\right|
$$

Since $\left|y_{i}-y_{j}\right| \lesssim h$, we have $\left|\left(M^{-1} \Theta M\right)_{i j}\right| \lesssim 1$ and the proof of the Lemma is complete.

Next result is an auxiliary estimate used later in the proof of Lemma 5.4 and 5.5. In the statement of the lemma we used the notation given at the end of Section 2 .

Proposition 5.3. Assume that $\theta \neq 0$ and convex in $K_{4}$. Then the following inequality holds

$$
\left\|\frac{\partial(\theta w)}{\partial x}\right\|_{0, K}^{2} \gtrsim|K|\left(\max _{E \in \partial K_{4}}\left\{\left|\delta_{E}^{K_{4}} y\right|\right\}^{2} \cdot\left(w_{E_{m i n}}^{K_{4}}\right)^{2}+\left(\delta_{E_{m i n}}^{K_{4}} y\right)^{2} \cdot \max _{E \in \partial K_{4}}\left\{w_{E}^{K_{4}}\right\}^{2}\right) .
$$

Proof. Let $E_{\min }$ be defined as (5.1). Without loss of generality, assume $E_{\min }=\left\{\left(x_{4}, y_{4}\right),\left(x_{5}, y_{5}\right)\right\}$, and then $w_{6}=w_{E_{m i n}}^{K_{4}}$. This means

$$
\left|y_{5}-y_{4}\right|=\min \left\{\left|y_{5}-y_{4}\right|,\left|y_{4}-y_{6}\right|,\left|y_{6}-y_{5}\right|\right\} \text {. }
$$

Hence

$$
\left|\frac{y_{5}-y_{4}}{y_{6}-y_{5}}\right| \leq 1, \quad\left|\frac{y_{5}-y_{4}}{y_{4}-y_{6}}\right| \leq 1
$$

and by triangle inequalities we have

$$
\left|\frac{y_{4}-y_{6}}{y_{6}-y_{5}}\right| \leq 2, \quad\left|\frac{y_{6}-y_{5}}{y_{4}-y_{6}}\right| \leq 2
$$

According to the expression (5.5) and the above inequalities, we have

$$
\begin{aligned}
\left\|\frac{\partial(\theta w)}{\partial x}\right\|_{0, K}^{2} \approx & \frac{1}{|K|}\left\{\left[\left(y_{4}-y_{6}\right) w_{6}+\left(y_{5}-y_{4}\right) w_{5}\right]^{2}+\left[\left(y_{5}-y_{4}\right) w_{4}+\left(y_{6}-y_{5}\right) w_{6}\right]^{2}\right. \\
& \left.\quad+\left[\left(y_{6}-y_{5}\right) w_{5}+\left(y_{4}-y_{6}\right) w_{4}\right]^{2}\right\} \\
& \gtrsim \frac{1}{|K|}\left\{\left[\left(y_{4}-y_{6}\right) w_{6}+\left(y_{5}-y_{4}\right) w_{5}\right]+\left[\left(y_{5}-y_{4}\right) w_{4}+\left(y_{6}-y_{5}\right) w_{6}\right] \frac{y_{4}-y_{6}}{y_{6}-y_{5}}\right. \\
& \left.\quad-\left[\left(y_{6}-y_{5}\right) w_{5}+\left(y_{4}-y_{6}\right) w_{4}\right] \frac{y_{5}-y_{4}}{y_{6}-y_{5}}\right\}^{2} \\
= & \frac{2}{|K|}\left[\left(y_{4}-y_{6}\right) w_{6}\right]^{2} \\
& \gtrsim \frac{1}{|K|} \max \left\{\left|y_{5}-y_{4}\right|,\left|y_{4}-y_{6}\right|,\left|y_{6}-y_{5}\right|\right\}^{2} w_{6}^{2} .
\end{aligned}
$$

Combining with (5.5), we have

$$
\left\|\frac{\partial(\theta w)}{\partial x}\right\|_{0, K}^{2} \gtrsim \frac{1}{|K|}\left\{\left[\left(y_{5}-y_{4}\right) w_{4}\right]^{2}+\left[\left(y_{5}-y_{4}\right) w_{5}\right]^{2}\right\} .
$$

So

$$
\left\|\frac{\partial(\theta w)}{\partial x}\right\|_{0, K}^{2} \gtrsim \frac{1}{|K|}\left\{\max \left\{\left|y_{5}-y_{4}\right|,\left|y_{4}-y_{6}\right|,\left|y_{6}-y_{5}\right|\right\}^{2} w_{6}^{2}+\left(y_{5}-y_{4}\right)^{2} w_{4}^{2}+\left(y_{5}-y_{4}\right)^{2} w_{5}^{2}\right\} .
$$

Notice again, here $E_{\min }=\left\{\left(x_{4}, y_{4}\right),\left(x_{5}, y_{5}\right)\right\}$ and $w_{6}=w_{E_{m i n}}^{K_{4}}$, then we get the result. 
We need to notice that till now we only require the mesh is quasi-uniform, since when $\theta$ is convex in element $K_{4}$, the semi-norm of interpolation function $\left\|\frac{\partial\left(I_{h}(\theta w)\right)}{\partial x}\right\|_{0, K}$ can be bounded by $\left\|\frac{\partial(\theta w)}{\partial x}\right\|_{0, K}$. However, this is not true when $\theta(y)$ is concave. In this case, $\left\|\frac{\partial\left(I_{h}(\theta w)\right)}{\partial x}\right\|_{0, K}$ may also depend on some neighboring element. To get the information of the neighboring element, we assume the partition $\mathcal{T}_{h}$ is uniform in the following.

Lemma 5.4. Assume that $\theta$ is nonzero at only one vertex of $K_{4}$ and that $K^{\prime}$ is the unique element from $\mathcal{T}_{H}$ which has this vertex on one of its edges. Assume also that $\theta$ is concave in $K_{4}$ (Case 2). Then the following inequality holds

$$
\left\|\frac{\partial\left(I_{h}(\theta w)\right)}{\partial x}\right\|_{0, K}^{2} \lesssim\left\|\frac{\partial(\theta w)}{\partial x}\right\|_{0, K^{\prime}}^{2} .
$$

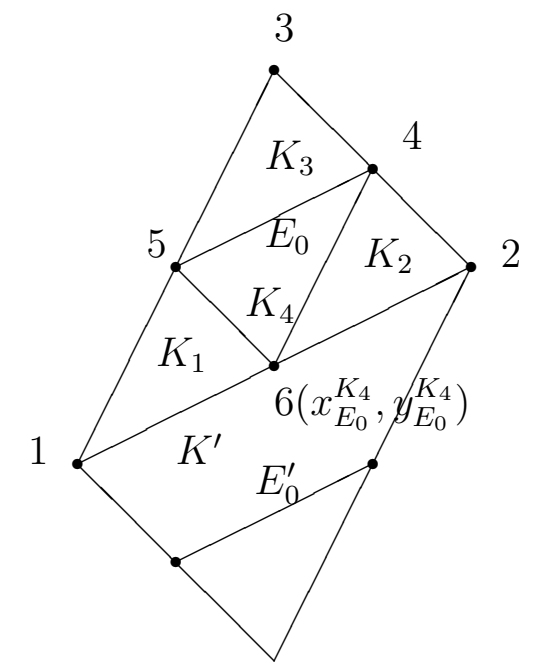

Figure 5.1. The coarse elements $K$ and $K^{\prime}$ sharing the same midpoint

Proof. Without loss of generality, assume $\theta_{E_{0}}^{K_{4}}$ is the only nonzero value. There are two possibilities: (a) $E_{0}=E_{\text {min }}$; and (b) $E_{0} \neq E_{\text {min }}$.

Proof in case $(\mathbf{a})$. Since $E_{0}=E_{m i n}$, we conclude that $\left|\delta_{E_{0}}^{K_{4}} y\right|=\min _{E \in \partial K_{4}}\left\{\left|\delta_{E}^{K_{4}} y\right|\right\}$.

We then have

$$
\begin{aligned}
\left\|\frac{\partial\left(I_{h}(\theta w)\right)}{\partial x}\right\|_{0, K}^{2} & \left.=\frac{|K|}{4} \sum_{E \in \partial K_{4}}\left|\delta_{E}^{K_{4}} y\right|^{2}\left(\theta_{E_{0}}^{K_{4}} w_{E_{0}}^{K_{4}}\right)^{2} \quad \text { (from 2.5) }\right) \\
& \lesssim|K| \max _{E \in \partial K_{4}}\left\{\left|\delta_{E}^{K_{4}} y\right|\right\}^{2}\left(w_{E_{0}}^{K_{4}}\right)^{2} .
\end{aligned}
$$

Since the partition $\mathcal{T}_{H}$ is uniform (see Figure 5.1), and $K^{\prime}$ is the element sharing the same point $\left(x_{E_{0}}^{K_{4}}, y_{E_{0}}^{K_{4}}\right)$ with $K$, we know that the values of $\theta$ at midpoints of $K^{\prime}$ are all nonzero. This is so, because the support of $\theta$, whose width is $2 h$ must include $K^{\prime}$ in its interior. Assume now that $E_{0}^{\prime}$ is the edge opposite to point $\left(x_{E_{0}}^{K_{4}}, y_{E_{0}}^{K_{4}}\right)$ in $K_{4}^{\prime}$ (i.e. $\left(x_{E_{0}}^{K_{4}}, y_{E_{0}}^{K_{4}}\right)=\left(x_{E_{0}^{\prime}}^{K_{4}^{\prime}}, y_{E_{0}^{\prime}}^{K_{4}^{\prime}}\right)$, see Figure 5.1. Observe that $E_{0}^{\prime}=E_{m i n}^{\prime}$ or $\left|\delta_{E_{0}^{\prime}}^{K_{4}^{\prime}} y\right|=\min _{E^{\prime} \in \partial K_{4}^{\prime}}\left\{\left|\delta_{E^{\prime}}^{K_{4}^{\prime}} y\right|\right\}$, because $E_{0}^{\prime}$ is a parallel 
translation of $E_{0}$. By Proposition 5.3, we now have

$$
\left\|\frac{\partial(\theta w)}{\partial x}\right\|_{0, K^{\prime}}^{2} \gtrsim\left|K^{\prime}\right| \max _{E^{\prime} \in \partial K_{4}^{\prime}}\left\{\left|\delta_{E^{\prime}}^{K_{4}^{\prime}} y\right|\right\}^{2}\left(w_{E_{0}^{\prime}}^{K_{4}^{\prime}}\right)^{2}=|K| \max _{E \in \partial K_{4}}\left\{\left|\delta_{E}^{K_{4}} y\right|\right\}^{2}\left(w_{E_{0}}^{K_{4}}\right)^{2} .
$$

So $\left\|\frac{\partial\left(I_{h}(\theta w)\right)}{\partial x}\right\|_{0, K}^{2} \lesssim\left\|\frac{\partial(\theta w)}{\partial x}\right\|_{0, K^{\prime}}^{2}$, and this completes the proof in case (a).

Proof in case (b). In case (b) we have $E_{0} \neq E_{\text {min }}$ and hence $\left|\delta_{E_{0}}^{K_{4}} y\right| \neq \min _{E \in \partial K_{4}}\left\{\left|\delta_{E}^{K_{4}} y\right|\right\}$. Since $\theta_{E_{0}}^{K_{4}}$ is the only nonzero value among the values of $\theta$ at the vertices of $K_{4}$, we easily get

$$
\theta_{E_{0}}^{K_{4}} \lesssim h^{-1} \min _{E \in \partial K_{4}}\left\{2\left|K_{4}\right| \delta_{E}^{K_{4}} y \mid\right\}
$$

Then

$$
\begin{aligned}
\left\|\frac{\partial\left(I_{h}(\theta w)\right)}{\partial x}\right\|_{0, K}^{2} & =\frac{|K|}{4} \sum_{E \in \partial K_{4}}\left|\delta_{E}^{K_{4}} y\right|^{2}\left(\theta_{E_{0}}^{K_{4}} w_{E_{0}}^{K_{4}}\right)^{2} \quad(\text { from }(2.5)) \\
& \lesssim|K| \max _{E \in \partial K_{4}}\left\{\left|\delta_{E}^{K_{4}} y\right|\right\}^{2}\left(\theta_{E_{0}}^{K_{4}}\right)^{2}\left(w_{E_{0}}^{K_{4}}\right)^{2} \\
& \lesssim|K| \max _{E \in \partial K_{4}}\left\{2\left|K_{4}\right| \delta_{E}^{K_{4}} y \mid\right\}^{2} h^{-2} \min _{E \in \partial K_{4}}\left\{\left|\delta_{E}^{K_{4}} y\right|\right\}^{2}\left(w_{E_{0}}^{K_{4}}\right)^{2} \\
& \lesssim|K| \min _{E \in \partial K_{4}}\left\{\left|\delta_{E}^{K_{4}} y\right|\right\}^{2}\left(w_{E_{0}}^{K_{4}}\right)^{2} .
\end{aligned}
$$

Let $K^{\prime}$ be the same as before, then by Proposition 5.3, we have

$$
\left\|\frac{\partial(\theta w)}{\partial x}\right\|_{0, K^{\prime}}^{2} \gtrsim\left|K^{\prime}\right| \min _{E^{\prime} \in \partial K_{4}^{\prime}}\left\{\left|\delta_{E^{\prime}}^{K_{4}^{\prime}} y\right|\right\}^{2}\left(w_{E_{0}^{\prime}}^{K_{4}^{\prime}}\right)^{2}=|K| \min _{E \in \partial K_{4}}\left\{\left|\delta_{E}^{K_{4}} y\right|\right\}^{2}\left(w_{E_{0}}^{K_{4}}\right)^{2} .
$$

Combining the last two inequalities then gives $\left\|\frac{\partial\left(I_{h}(\theta w)\right)}{\partial x}\right\|_{0, K}^{2} \lesssim\left\|\frac{\partial(\theta w)}{\partial x}\right\|_{0, K^{\prime}}^{2}$. This completes the proof in case (b), and also the proof of the Lemma.

The next Lemma gives the stability estimates in the last case (Case 3) and we refer to Figure 5.2 for clarifying the notation.

Lemma 5.5. Assume that $\theta$ is nonzero at exactly two vertices of $K_{4}$ and concave in $K_{4}$ (Case 3). Let $K^{\prime}$ be an element from $\mathcal{T}_{H}$ which shares with $K_{4}$ the vertex at which $\theta$ has larger value on one of its edges. Then the following inequality holds

$$
\left\|\frac{\partial\left(I_{h}(\theta w)\right)}{\partial x}\right\|_{0, K}^{2} \lesssim\left\|\frac{\partial(\theta w)}{\partial x}\right\|_{0, K \cup K^{\prime}}^{2}
$$

Proof. Without loss of generality we may assume that $\theta_{4}$ and $\theta_{5}$ are the only nonzero values of $\theta$, we may also assume that $\theta_{4} \geq \theta_{5}$. As a consequence, $K^{\prime}$ will share $\left(x_{4}, y_{4}\right)$ with $K$.

We consider two possibilities: (a) $E_{\min }=\left\{\left(x_{4}, y_{4}\right),\left(x_{5}, y_{5}\right)\right\}$; (b) $E_{\min } \neq\left\{\left(x_{4}, y_{4}\right),\left(x_{5}, y_{5}\right)\right\}$.

Proof of (a). Since $E_{\min }=\left\{\left(x_{4}, y_{4}\right),\left(x_{5}, y_{5}\right)\right\}$, we have that $\left|y_{5}-y_{4}\right|=\min \left\{\left|y_{5}-y_{4}\right|, \mid y_{4}-\right.$ $\left.y_{6}|,| y_{6}-y_{5} \mid\right\}$. Hence, from 2.5) we obtain

$$
\begin{array}{r}
\left\|\frac{\partial\left(I_{h}(\theta w)\right)}{\partial x}\right\|_{0, K}^{2} \approx \frac{1}{|K|}\left\{\left(\left(y_{5}-y_{4}\right) \theta_{4} w_{4}\right)^{2}+\left(\left(y_{5}-y_{4}\right) \theta_{5} w_{5}\right)^{2}\right. \\
\left.+\left(\left(y_{6}-y_{5}\right) \theta_{5} w_{5}+\left(y_{4}-y_{6}\right) \theta_{4} w_{4}\right)^{2}\right\} .
\end{array}
$$




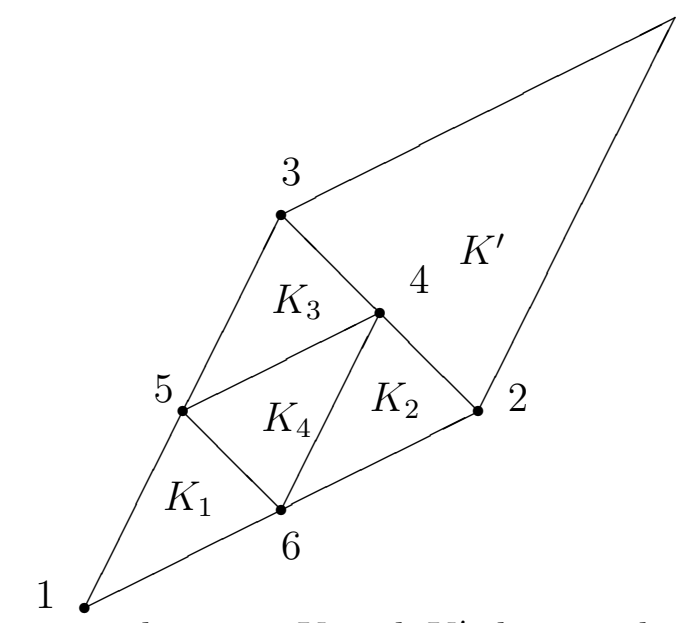

Figure 5.2. The coarse elements $K$ and $K^{\prime}$ sharing the same midpoint

We want to bound now all the terms on the right side of the above relation with quantities independent of the values of $\theta$. From the fact that $\theta \leq 1$ we have that

$$
\left(\left(y_{5}-y_{4}\right) \theta_{4} w_{4}\right)^{2} \lesssim\left(\left(y_{5}-y_{4}\right) w_{4}\right)^{2} .
$$

The other terms are bounded as follows

$$
\begin{aligned}
\left(\left(y_{5}-y_{4}\right) \theta_{5} w_{5}\right)^{2} & \leq\left(\left(y_{5}-y_{4}\right) w_{5}\right)^{2} \lesssim\left(\left(y_{5}-y_{4}\right) w_{5}\right)^{2}\left(\frac{y_{6}-y_{5}}{y_{4}-y_{6}}\right)^{2} \\
& =\left(\left(\left(y_{6}-y_{5}\right) w_{5}+\left(y_{4}-y_{6}\right) w_{4}\right) \frac{y_{5}-y_{4}}{y_{4}-y_{6}}-\left(y_{5}-y_{4}\right) w_{4}\right)^{2} \\
& \lesssim\left(\left(y_{6}-y_{5}\right) w_{5}+\left(y_{4}-y_{6}\right) w_{4}\right)^{2}\left(\frac{y_{5}-y_{4}}{y_{4}-y_{6}}\right)^{2}+\left(\left(y_{5}-y_{4}\right) w_{4}\right)^{2} \\
& \lesssim\left(\left(y_{6}-y_{5}\right) w_{5}+\left(y_{4}-y_{6}\right) w_{4}\right)^{2}+\left(\left(y_{5}-y_{4}\right) w_{4}\right)^{2}
\end{aligned}
$$

and also

$$
\begin{aligned}
\left(\left(y_{6}-y_{5}\right) \theta_{5} w_{5}+\left(y_{4}-y_{6}\right) \theta_{4} w_{4}\right)^{2} & =\left(\left(y_{6}-y_{5}\right) \theta_{5} w_{5}+\left(y_{4}-y_{6}\right) \theta_{5} w_{4}-\left(y_{4}-y_{6}\right)\left(\theta_{5}-\theta_{4}\right) w_{4}\right)^{2} \\
& =\left(\left(\left(y_{6}-y_{5}\right) w_{5}+\left(y_{4}-y_{6}\right) w_{4}\right) \theta_{5}-\left(y_{4}-y_{6}\right) w_{4} \frac{y_{5}-y_{4}}{h}\right)^{2} \\
& \lesssim\left(\left(y_{6}-y_{5}\right) w_{5}+\left(y_{4}-y_{6}\right) w_{4}\right)^{2}+\left(\left(y_{5}-y_{4}\right) w_{4}\right)^{2}
\end{aligned}
$$

Hence we get

$$
\left\|\frac{\partial\left(I_{h}(\theta w)\right)}{\partial x}\right\|_{0, K}^{2} \lesssim \frac{1}{|K|}\left\{\left(\left(y_{5}-y_{4}\right) w_{4}\right)^{2}+\left(\left(y_{6}-y_{5}\right) w_{5}+\left(y_{4}-y_{6}\right) w_{4}\right)^{2}\right\} .
$$

In this case,

$$
\left\|\frac{\partial(\theta w)}{\partial x}\right\|_{0, K}^{2} \gtrsim \frac{1}{|K|}\left\{\left(\left(y_{6}-y_{5}\right) w_{5}+\left(y_{4}-y_{6}\right) w_{4}\right)^{2}\right\}
$$


Since $K^{\prime}$ denotes the element sharing $\left(x_{4}, y_{4}\right)$ with $K$, we obtain

$$
\begin{aligned}
\left\|\frac{\partial(\theta w)}{\partial x}\right\|_{0, K^{\prime}}^{2} & \gtrsim \frac{1}{\left|K^{\prime}\right|}\left\{\min \left\{\left|y_{5}^{K^{\prime}}-y_{4}^{K^{\prime}}\right|,\left|y_{4}^{K^{\prime}}-y_{6}^{K^{\prime}}\right|,\left|y_{6}^{K^{\prime}}-y_{5}^{K^{\prime}}\right|\right\}^{2} w_{4}^{2}\right\} \\
& =\frac{1}{|K|}\left\{\min \left\{\left|y_{5}-y_{4}\right|,\left|y_{4}-y_{6}\right|,\left|y_{6}-y_{5}\right|\right\}^{2} w_{4}^{2}\right\} \\
& =\frac{1}{|K|}\left\{\left(y_{5}-y_{4}\right)^{2} w_{4}^{2}\right\},
\end{aligned}
$$

SO

$$
\left\|\frac{\partial(\theta w)}{\partial x}\right\|_{0, K \cup K^{\prime}}^{2} \gtrsim \frac{1}{|K|}\left\{\left(\left(y_{5}-y_{4}\right) w_{4}\right)^{2}+\left(\left(y_{6}-y_{5}\right) w_{5}+\left(y_{4}-y_{6}\right) w_{4}\right)^{2}\right\} \gtrsim\left\|\frac{\partial\left(I_{h}(\theta w)\right)}{\partial x}\right\|_{0, K}^{2} .
$$

This completes the proof in case (a).

Proof of (b). In this case we have that $E_{\text {min }} \neq\left\{\left(x_{4}, y_{4}\right),\left(x_{5}, y_{5}\right)\right\}$, which is equivalent to $\left|y_{5}-y_{4}\right|>\min \left\{\left|y_{5}-y_{4}\right|,\left|y_{4}-y_{6}\right|,\left|y_{6}-y_{5}\right|\right\}$.

Since $\theta_{4}>\theta_{5}\left(\theta_{4}=\theta_{5}\right.$ can not be true in this case, because $\theta_{4}=\theta_{5}$ implies $\left.y_{4}=y_{5}\right)$, we can get $\left|y_{6}-y_{5}\right|<\left|y_{4}-y_{6}\right|$. It is then easy to see $\left|y_{6}-y_{5}\right|=\min \left\{\left|y_{5}-y_{4}\right|,\left|y_{4}-y_{6}\right|,\left|y_{6}-y_{5}\right|\right\}$. Then

$$
\theta_{5} \lesssim h^{-1} \min \left\{\left|y_{5}-y_{4}\right|,\left|y_{4}-y_{6}\right|,\left|y_{6}-y_{5}\right|\right\}=h^{-1}\left|y_{6}-y_{5}\right|
$$

So from (2.5), we have

$$
\begin{aligned}
\left\|\frac{\partial\left(I_{h}(\theta w)\right)}{\partial x}\right\|_{0, K}^{2} & \approx \frac{1}{|K|}\left\{\left(\left(y_{5}-y_{4}\right) \theta_{5} w_{5}\right)^{2}+\left(\left(y_{5}-y_{4}\right) \theta_{4} w_{4}\right)^{2}+\left(\left(y_{6}-y_{5}\right) \theta_{5} w_{5}+\left(y_{4}-y_{6}\right) \theta_{4} w_{4}\right)^{2}\right\} \\
& \lesssim \frac{1}{|K|}\left\{\left(\left(y_{5}-y_{4}\right) \theta_{5} w_{5}\right)^{2}+\left(\left(y_{5}-y_{4}\right) \theta_{4} w_{4}\right)^{2}+\left(\left(y_{6}-y_{5}\right) \theta_{5} w_{5}\right)^{2}+\left(\left(y_{4}-y_{6}\right) \theta_{4} w_{4}\right)^{2}\right\} \\
& \lesssim \frac{1}{|K|}\left\{\left(\left(y_{6}-y_{5}\right) w_{5}\right)^{2}+\left(\left(y_{4}-y_{6}\right) w_{4}\right)^{2}\right\} .
\end{aligned}
$$

In this case,

$$
\left\|\frac{\partial(\theta w)}{\partial x}\right\|_{0, K}^{2} \gtrsim \frac{1}{|K|}\left\{\left(\left(y_{6}-y_{5}\right) w_{5}+\left(y_{4}-y_{6}\right) w_{4}\right)^{2}\right\} .
$$

Since the partition $\mathcal{T}_{H}$ is uniform (see Figure 5.2), and $K^{\prime}$ is the element sharing the same point $\left(x_{4}, y_{4}\right)$ with $K$, we know that the values of $\theta$ at midpoints of $K^{\prime}$ are all nonzero. Observe that the edge opposite to point $\left(x_{4}, y_{4}\right)$ in $K_{4}^{\prime}$ is a parallel translation of the edge opposite to point $\left(x_{4}, y_{4}\right)$ in $K_{4}$. That is to say, point $\left(x_{4}, y_{4}\right)$ in $K^{\prime}$ (also point 4 in $K$ ) is just the midpoint opposite to edge with $\min \left\{\left|y_{5}^{K^{\prime}}-y_{4}^{K^{\prime}}\right|,\left|y_{4}^{K^{\prime}}-y_{6}^{K^{\prime}}\right|,\left|y_{6}^{K^{\prime}}-y_{5}^{K^{\prime}}\right|\right\}$. By Proposition 5.3, we have

$$
\begin{aligned}
\left\|\frac{\partial(\theta w)}{\partial x}\right\|_{0, K^{\prime}}^{2} & \gtrsim \frac{1}{\left|K^{\prime}\right|}\left\{\max \left\{\left|y_{5}^{K^{\prime}}-y_{4}^{K^{\prime}}\right|,\left|y_{4}^{K^{\prime}}-y_{6}^{K^{\prime}}\right|,\left|y_{6}^{K^{\prime}}-y_{5}^{K^{\prime}}\right|\right\}^{2} w_{4}^{2}\right\} \\
& =\frac{1}{|K|}\left\{\max \left\{\left|y_{5}-y_{4}\right|,\left|y_{4}-y_{6}\right|,\left|y_{6}-y_{5}\right|\right\}^{2} w_{4}^{2}\right\} .
\end{aligned}
$$

Combining the last two inequalities, we have 


$$
\begin{aligned}
\left\|\frac{\partial(\theta w)}{\partial x}\right\|_{0, K \cup K^{\prime}}^{2} & \gtrsim \frac{1}{|K|}\left\{\left(\left(y_{6}-y_{5}\right) w_{5}+\left(y_{4}-y_{6}\right) w_{4}\right)^{2}+\max \left\{\left|y_{5}-y_{4}\right|,\left|y_{4}-y_{6}\right|,\left|y_{6}-y_{5}\right|\right\}^{2} w_{4}^{2}\right\} \\
& \gtrsim \frac{1}{|K|}\left\{\left(\left(y_{6}-y_{5}\right) w_{5}\right)^{2}+\left(\left(y_{4}-y_{6}\right) w_{4}\right)^{2}\right\} \\
& \gtrsim\left\|\frac{\partial\left(I_{h}(\theta w)\right)}{\partial x}\right\|_{0, K}^{2} .
\end{aligned}
$$

This completes the proof of case (b), and also the proof of Lemma.

Lemma 5.6. For any $w \in V_{h}$, if $I_{H} w=0$, then for any $1 \leq i \leq L$,

$$
\left\|\frac{\partial\left(I_{h}\left(\theta_{i} w\right)\right)}{\partial x}\right\|_{0}^{2} \lesssim\left\|\frac{\partial\left(\theta_{i} w\right)}{\partial x}\right\|_{0}^{2}
$$

Proof. The estimate follows from the local (element-wise) estimates given by Lemma 5.2 , 5.4, 5.5, and summation over all elements from $\mathcal{T}_{H}$.

\section{Proof of the theorem 3.4}

In this section we prove the convergence result that we have already stated in Section 3 .

Theorem 3.4. For any angle of rotation $\omega \in[0, \pi]$, the two-level iteration with coarse space $V_{H}$ and line (block) Gauss-Seidel smoother is a uniformly convergent method. In fact, we have

$$
\left\|E_{T L}\right\|_{a}^{2} \leq 1-\frac{1}{C}
$$

with constant $C$ independent of $\epsilon$ and $h$.

Proof. From Lemma 3.2 , we only need to prove $\sup _{v \in V_{h}} \frac{\left\|\left(I-\Pi_{*}\right) v\right\|_{*}^{2}}{\|v\|_{a}^{2}} \leq C$.

For any $v \in V_{h}$, let $w:=v-I_{H} v, w_{i}:=I_{h}\left(\theta_{i}(y) w\right)$, it is easy to see $I_{H} w=0$, and

$$
\sum_{i} w_{i}=\sum_{i} I_{h}\left(\theta_{i}(y) w\right)=I_{h} \sum_{i}\left(\theta_{i}(y) w\right)=I_{h} w=w .
$$

Then

$$
\begin{aligned}
\sup _{v \in V_{h}} \frac{\left\|\left(I-\Pi_{*}\right) v\right\|_{*}^{2}}{\|v\|_{a}^{2}} & \leq \sup _{v \in V_{h}} \frac{\left\|\left(I-I_{H}\right) v\right\|_{*}^{2}}{\|v\|_{a}^{2}} \\
& =\sup _{v \in V_{h}} \frac{\|w\|_{*}^{2}}{\|v\|_{a}^{2}}=\sup _{v \in V_{h}} \inf _{\sum_{i} \tilde{w}_{i}=w} \frac{\sum_{i}\left\|\tilde{w}_{i}\right\|_{a}^{2}}{\|v\|_{a}^{2}} \leq \sup _{v \in V_{h}} \frac{\sum_{i}\left\|w_{i}\right\|_{a}^{2}}{\|v\|_{a}^{2}} .
\end{aligned}
$$

So we only need to prove for any $v \in V_{h}, \sum_{i}\left\|w_{i}\right\|_{a}^{2} \lesssim\|v\|_{a}^{2}$.

First, the decomposition is stable in $L^{2}$,

$$
\sum_{i}\left\|w_{i}\right\|_{0}^{2} \lesssim\left\|\sum_{i} w_{i}\right\|_{0}^{2}=\|w\|_{0}^{2} \lesssim \sum_{i}\left\|w_{i}\right\|_{0}^{2}
$$


Since the sum is along $x$ direction,

$$
\begin{aligned}
\sum_{i}\left\|\partial_{x} w_{i}\right\|_{0}^{2} & =\sum_{i}\left\|\partial_{x}\left(I_{h}\left(\theta_{i}(y) w\right)\right)\right\|_{0}^{2} \\
& \lesssim \sum_{i}\left\|\partial_{x}\left(\theta_{i}(y) w\right)\right\|_{0}^{2} \quad \text { (by Lemma 5.6) } \\
& =\sum_{i}\left\|\theta_{i}(y) \partial_{x} w\right\|_{0}^{2} \\
& =\sum_{i}\left\|\theta_{i}(y)\right\|^{2}\left\|\partial_{x} w\right\|_{0}^{2} \\
& \lesssim\left\|\partial_{x} w\right\|_{0}^{2}
\end{aligned}
$$

then

$$
\begin{aligned}
\sum_{i}\left\|w_{i}\right\|_{a}^{2} & =\sum_{i}\left\|\partial_{x} w_{i}\right\|_{0}^{2}+\sum_{i} \epsilon\left\|\partial_{y} w_{i}\right\|_{0}^{2} \\
& \lesssim \sum_{i}\left\|\partial_{x} w_{i}\right\|_{0}^{2}+\sum_{i} \epsilon h^{-2}\left\|w_{i}\right\|_{0}^{2} \quad \text { (by inverse inequality) } \\
& \lesssim\left\|\partial_{x} w\right\|_{0}^{2}+\epsilon h^{-2}\|w\|_{0}^{2} \quad(\text { by }(6.1) \text { and }(6.2)) \\
& =\left\|\partial_{x}\left(v-I_{H} v\right)\right\|_{0}^{2}+\epsilon h^{-2}\left\|v-I_{H} v\right\|_{0}^{2} \\
& \lesssim\left\|\partial_{x} v\right\|_{0}^{2}+\epsilon h^{-2} h^{2}|v|_{1}^{2} \quad(\text { by Lemma } 4.3) \\
& \lesssim|v|_{a}^{2} .
\end{aligned}
$$

\section{Numerical Experiments}

7.1. Tests for two-level method on a rotated uniform mesh. We first test the performance of the two-level iterative method and its convergence properties with respect to $\epsilon$ and $h$. We pick as initial triangulation a $4 \times 4$ mesh with a characteristic mesh size $h_{0}=\frac{1}{2} \sqrt{2}$ as shown in Figure 2.1. We then apply the two-level method described earlier on sequence of meshes with mesh sizes $h_{k}=2^{-k} h_{0}, k=1, \ldots, 6$.

The energy norm of the error of two-level method $\left\|E_{T L}\right\|_{a}$ is depicted in Figure 7.1 show that two-level method is uniformly convergent w.r.t. $\epsilon$ and $h$, which agree with the theoretical results we have proved in the previous sections.

7.2. Tests for two-level method on a general unstructured mesh. Similarly to the case of uniform mesh, for a general unstructured mesh we choose $h_{0}=0.9$ as the maximum diameter of the triangles on the coarsest mesh $\mathcal{T}_{0}$ as shown in Figure 7.2. This coarsest mesh is then refined 6 times and get the mesh to obtain a sequence of triangulations with characteristic mesh sizes $h=2^{-k} h_{0}, k=1, \ldots, 6$.

The energy norm of the error propagation operator for the two level method, $\left\|E_{T L}\right\|_{a}$, are shown in Figure 7.3. The uniform convergence is clearly seen from the plots. Theoretical justification of such uniform convergence is however much more difficult and is a topic of current and future research. 


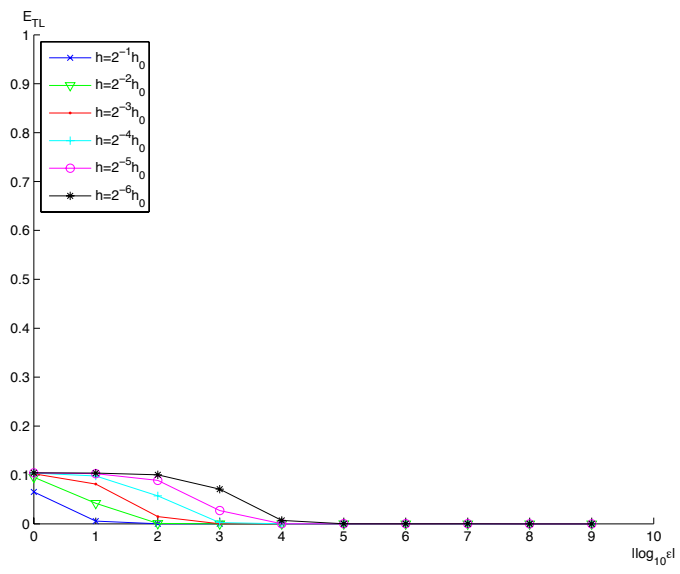

(a) $\omega=0$

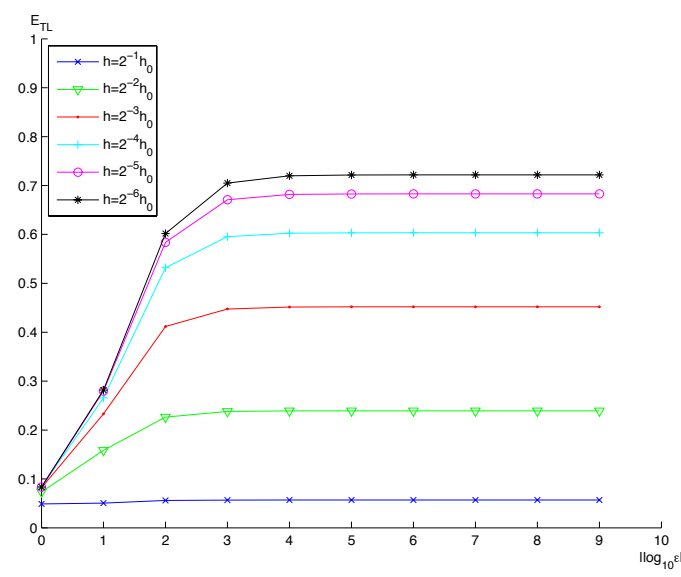

(b) $\omega=\pi / 6$

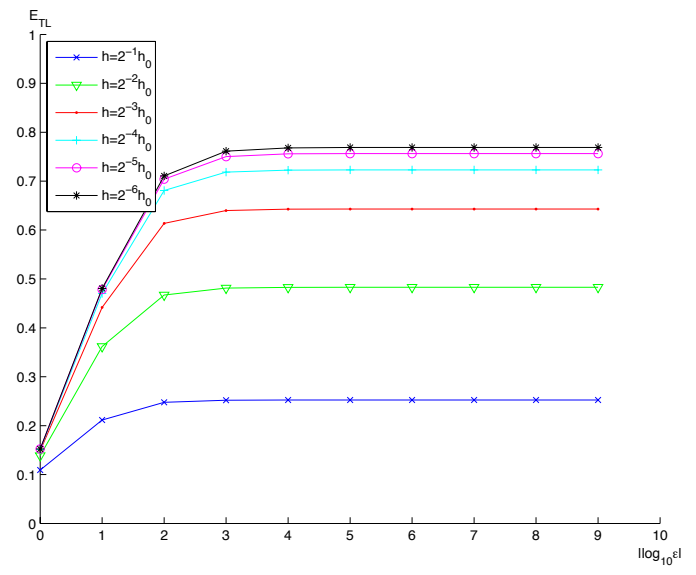

(c) $\omega=\pi / 4$

Figure 7.1. Plot of the convergence rates $\left\|E_{T L}\right\|_{a}^{2}$ versus $\log _{10} \epsilon$ for a sequence of uniform meshes with varying angle of anisotropy
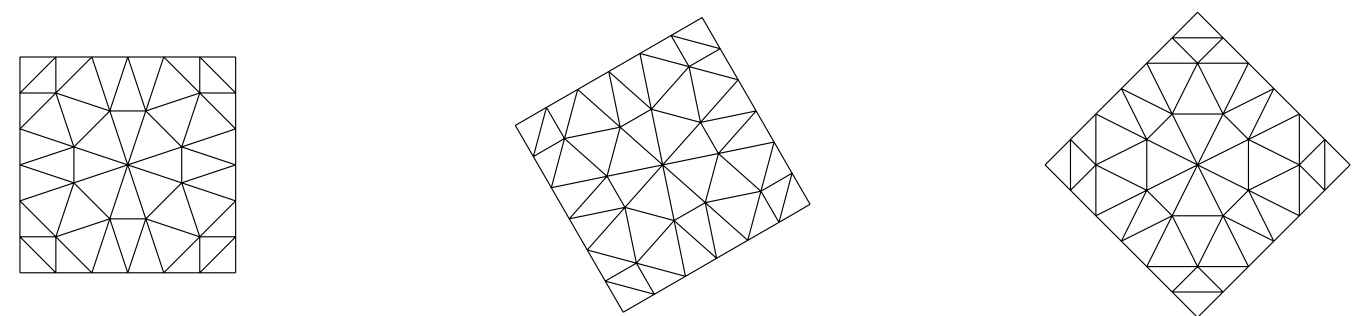

Figure 7.2. Plot of unstructured grids used in the numerical examples for three values of the angle of rotation of anisotropy $\omega=0, \omega=\pi / 6$ and $\omega=\pi / 4$.

\section{REFERENCES}

[1] Rob Stevenson. New estimates of the contraction number of $V$-cycle multi-grid with applications to anisotropic equations. In Incomplete decomposition (ILU) - algorithms, theory and applications (Kiel, 1992), volume 41 of Notes Numer. Fluid Mech., pages 159-167. Vieweg, Braunschweig, 1993. 


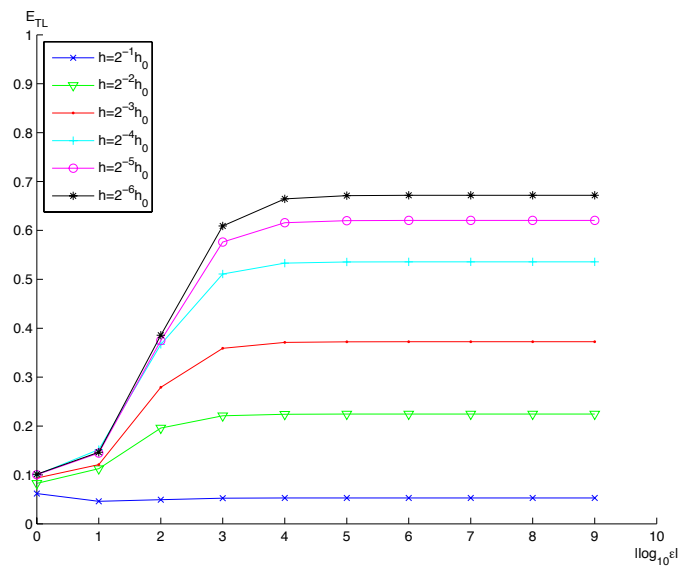

(a) $\omega=0$

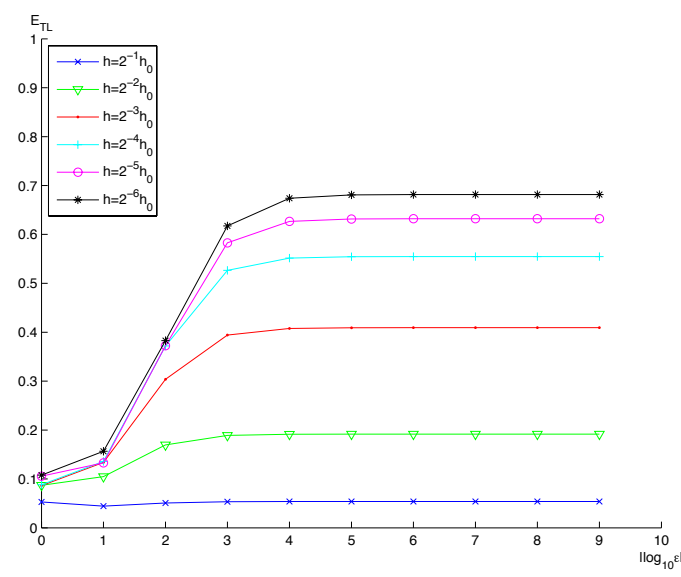

(b) $\omega=\pi / 6$

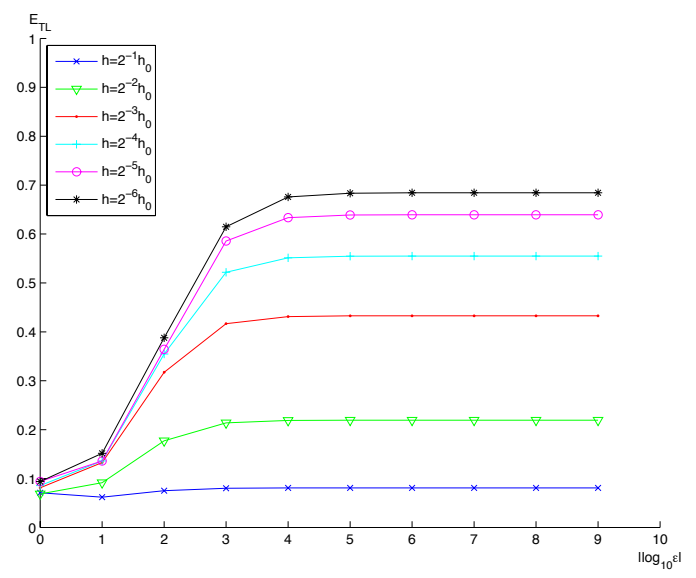

(c) $\omega=\pi / 4$

Figure 7.3. Plot of the convergence rates $\left\|E_{T L}\right\|_{a}^{2}$ versus $\log _{10} \epsilon$ for a sequence of refined general unstructured meshes with varying angle of anisotropy

[2] Rob Stevenson. Robust multi-grid with 7-point ILU smoothing. In Multigrid methods, IV (Amsterdam, 1993), volume 116 of Internat. Ser. Numer. Math., pages 295-307. Birkhäuser, Basel, 1994.

[3] Wolfgang Hackbusch. Multigrid methods and applications, volume 4 of Springer Series in Computational Mathematics. Springer-Verlag, Berlin, 1985.

[4] James H. Bramble and Xuejun Zhang. Uniform convergence of the multigrid $V$-cycle for an anisotropic problem. Math. Comp., 70(234):453-470, 2001.

[5] James H. Bramble, Joseph E. Pasciak, Jun Ping Wang, and Jinchao Xu. Convergence estimates for multigrid algorithms without regularity assumptions. Math. Comp., 57(195):23-45, 1991.

[6] Jinchao Xu. Iterative methods by space decomposition and subspace correction. SIAM Rev., 34(4):581613, 1992.

[7] Nicolas Neuss. $V$-cycle convergence with unsymmetric smoothers and application to an anisotropic model problem. SIAM J. Numer. Anal., 35(3):1201-1212 (electronic), 1998.

[8] Yongke Wu, Long Chen, Xiaoping Xie, and Jinchao Xu. Convergence analysis of v-cycle multigrid method for anisotropic elliptic equation. Finished.

[9] M. Griebel and P. Oswald. Tensor product type subspace splittings and multilevel iterative methods for anisotropic problems. Adv. Comput. Math., 4(1-2):171-206, 1995.

[10] Young-Ju Lee, Jinbiao Wu, Jinchao Xu, and Ludmil Zikatanov. Robust subspace correction methods for nearly singular systems. Math. Models Methods Appl. Sci., 17(11):1937-1963, 2007. 
[11] Ludmil T. Zikatanov. Two-sided bounds on the convergence rate of two-level methods. Numer. Linear Algebra Appl., 15(5):439-454, 2008.

[12] James H. Bramble and Jinchao Xu. Some estimates for a weighted $L^{2}$ projection. Math. Comp., 56(194):463-476, 1991.

School of Mathematics, Sichuan University, Chengdu, Sichuan, China, 610064

E-mail address: yuguozhumail@yahoo.com.cn

Department of Mathematics, Pennsylvania State University, University Park, State ColLEGE PA 16802

E-mail address: xu@math.psu.edu

Department of Mathematics, Pennsylvania State University, University Park, State ColLEGE PA 16802

E-mail address: 1tz@math.psu.edu 